\title{
Michel theory of symmetry breaking and gauge theories
}

\author{
Giuseppe Gaeta* \\ I.H.E.S., 35 Route de Chartres \\ 91440 Bures sur Yvette (France) \\ gaeta@ihes.fr \\ Paola Morando \\ Dipartimento di Matematica, Politecnico di Torino, \\ Corso Duca degli Abruzzi 24, 10129 Torino (Italy) \\ morando@polito.it
}

\begin{abstract}
Summary. We extend Michel's theorem on the geometry of symmetry breaking [1] to the case of pure gauge theories, i.e. of gauge-invariant functionals defined on the space $\mathcal{C}$ of connections of a principal fiber bundle. Our proof follows closely the original one by Michel, using several known results on the geometry of $\mathcal{C}$. The result (and proof) is also extended to the case of gauge theories with matter fields.
\end{abstract}

* On leave from Dept. of Mathematics, Loughborough University, Loughborough LE11 3TU (GB)

$27 / 1 / 97$

Research supported by the Volkswagen Stiftung under the RiP program at Oberwolfach. 


\section{Introduction}

In 1971, Louis Michel - motivated by the $S U(3)$ theory of hadronic interactions - proved a remarkable theorem on symmetry breaking in theories described by a $G$-invariant potential ( $G$ a compact semisimple Lie group) over a finite dimensional smooth $G$-manifold $M$ [1]; this result was a direct generalization of the theory he and Radicati had developed to study the geometry of the $S U(3)$ octet, and the model-independent features of the $S U(3)$ theory $[2-4]$.

Essentially, Michel theorem guarantees that, under suitable conditions, there are points in $M$ which are critical for any $G$-invariant potential $V: M \rightarrow \mathcal{R}$; moreover, these points are characterized in terms of a geometric construction which takes into account the symmetry properties of points - and subsets - of $M$ under the $G$ action. Notice that actually, critical points of $G$-invariant functions come necessarily in $G$-orbits; thus, whenever the orbit space $\Omega=M / G$ is well defined - as it is under the conditions mentioned above - it is convenient to set the problem directly in $\Omega$ (maybe with an explict use of the basis of $G$-invariant functions on $M$ as coordinates in $\Omega$ ), as Michel did.

Thus, in summary, the Michel theorem on critical orbits of $G$-invariant functions on a $G$-manifold [1] allows to identify $G$-orbits which are critical for any $G$-invariant function; thus, it permits to study spontaneous symmetry breaking in a model-independent way [3-16]; this theory has also been extended to study supersymmetry breaking [12]. Mathematical foundations for the theory are provided e.g. by $[17,18]$.

The purpose of the present note is to show that Michel's theory can be extended to the study of (pure) gauge theories, such as those defined by a Yang-Mills functional, and more generally by a gauge-invariant functional defined on a space of connections for a given principal bundle.

It will turn out that once this is obtained, extension to the case of complete gauge theories, i.e. theories with matter fields, follows rather easily ${ }^{1}$.

It should be stressed that, for our result to be of physical interest, the space of connections to be considered (see below) should obey some natural conditions. Thus, on the one side we would be not justified in restricting our attention to the (dense) subspace of irreducible connections, for which a full geometric description is well known $[22,23]$, and which is relatively simple (we recall however that in the $S U(2)$ case this coincides with the full space of connections [24]). On the other side, we should not consider the full space of connections, but only those connections satisfying a finite energy condition, or

1 In this respect, it should be mentioned that an extension of Michel theory to gauge functionals (in that case, dealing only with matter fields) was already considered in [19-21], but this suffered from several - strong - limitations; these followed from focusing on the seemingly easier sector of matter fields, and it turns out that the present approach is not only more natural geometrically, but also more fruitful. 
more precisely the completion of this set in suitable norm; this leads naturally to consider Sobolev spaces of connections and Sobolev norms [24,25]. The same remark would apply when considering sections of a vector bundle in the context of gauge theories with matter fields, see below.

In the present note, we will give a careful statement and a complete proof of the extension of Michel's theorem to pure gauge theories; we will also shortly discuss some - easy but relevant - extensions and generalizations of this (including the one to theories with matter fields), whose proof is only sketched or omitted at all as it would just repeat the one for the pure gauge case, and some of the possible applications.

It is appropriate to stress here that it turns out - as it will be clear in our discussion - that one can directly extend the essence of Michel's construction and proof, from its original setting $-M$ a finite dimensional manifold, $G$ a compact Lie group, in the following referred to as the "classical case" - to the case of gauge theories, which involve infinite dimensional, non-compact manifold and group (a large part of the results needed for this are classical ones $[22,23,26]$ ); this shows (see also the final discussion in sect.7) how far-reaching is the Michel theory of symmetry breaking.

Plan of the paper. Let us shortly describe the pan of the paper. We will start by collecting in sect.1 some well known geometrical fact to provide a proper setting - and fix notation - for the discussion to follow. In sect.2 we will define a Sobolev norm on the space of connections and on gauge sections, and choose some Sobolev class of connections - and correspondingly of gauge sections - in which we work; this is needed in order to deal with Hilbert spaces. With this, we can use known results concerning existence of slices. The central result of this note is given in sect.3; it is a direct extension of Michel's theorem [1] - and proof - to the pure gauge case; after this, we can pass to consider extension and consequences of it. Thus, first of all we notice in sect.4 that once we have the result for the pure gauge case, it can be easily extended to the case of gauge theories with matter fields. Then, in sect.5 we discuss some other extensions, dealing with general systems (i.e. not necessarily variational ones) with a gauge symmetry, and we formulate the gauge equivalent of reduction lemmata which are well known in the finite dimensional case; these are applied in sect. 6 to discuss specifically the problem of symmetry breaking in theories with a (continuous) dependence on a parameter. Finally, in sect.7 we discuss some further extensions, as well as the strength - and weakness - of the approach proposed here. The discussion of sects.5-7 refers only to the pure gauge case for ease of notation, but it will be clear from sect. 4 that these would also extend to the case where matter fields are present. All our discussion will be conducted using only the geometry of the problem, i.e. with no reference to the kind of specific functional one encounters in physical examples of gauge theories. To discuss these is obviously a very relevant matter, so that we do indeed consider them, and how our main theorem can be of use in determining specific solutions, in the appendices. Here we restrict specifically to the well known class of functionals made of the Yang-Mills one $L=\left|F_{\mu \nu}\right|^{2}$ for the pure gauge part, and by the standard Lagrangian $L=\left[|\nabla \phi|^{2}-V(\phi)\right]$ (see below) for the matter fields (with $\nabla$ the covariant derivative). In appendix A we study of special class of solutions, in appendix B we further restrict this 
to the case associated to the null connection $A^{0}$ (see below), and finally in appendix $\mathrm{C}$ we deal with the case $G=S U(3)$.

\section{Geometrical setting}

Let $B$ be an $n$-dimensional ( $n$ finite) riemannian manifold (e.g. $B=R^{4}$ or $S^{4}$ ); let $G$ be a compact, connected and semisimple Lie group (e.g. $G=S U(N)$ ); let $P$ be a principal fiber bundle over $B$ with projection $\pi: P \rightarrow B$ and fiber $\pi^{-1}(x) \approx G$. We denote by $\mathcal{G}$ the Lie algebra of $G$.

Let $\mathcal{C}$ be the set of connections on $P$. It is well known that $\mathcal{C}$ is an affine space modelled on the vector space $\mathcal{A}:=\Lambda^{1}\left(\mathrm{~T}^{*} B, \mathcal{G}\right)$ of the one-forms on $B$ taking value in $\mathcal{G}$. With any connection $A \in \mathcal{C}$ we can associate a covariant derivative $\nabla^{\alpha}$ and a one-form $\alpha \in \mathcal{A}$ that are given, in local coordinates $\left(x^{1}, \ldots, x^{n}\right)$ on $B$ and with $A_{\mu}: B \rightarrow \mathcal{G}$, by

$$
\begin{gathered}
\alpha=A_{\mu}(x) \mathrm{d} x^{\mu} ; \\
\nabla_{\mu}^{\alpha}=\partial_{\mu}+A_{\mu} .
\end{gathered}
$$

Choosing as reference point in $\mathcal{C}$ the null connection $A^{0}$ such that $A_{\mu}^{0}=0(\mu=1, \ldots, n)$, from now on we will say "the connection $\alpha$ " to mean "the connection $A=A^{0}+\alpha$ whose associated connection form is $\alpha$ ".

Let $\Gamma$ denote the space of differentiable sections of $P$; $\Gamma$ has the structure of a Hilbert-Lie group, and a section $\gamma \in \Gamma$ will be written in local coordinates as

$$
\gamma=g(x)
$$

with $g: B \rightarrow G$. We say that $\Gamma$ is a gauge group modelled on $G$.

The gauge group $\Gamma$ acts naturally on the operator of covariant derivative associated to a connection $A \in \mathcal{C}$ by conjugation, i.e. $\gamma: \nabla^{\alpha} \rightarrow \gamma \nabla^{\alpha} \gamma^{-1}$; the action of $\Gamma$ on $\mathcal{A}$ is given in local coordinates by

$$
\gamma: A_{\mu} \Longrightarrow g(x) \cdot A_{\mu}(x) \cdot g^{-1}(x)-\left(\partial_{\mu} g\right)(x) \cdot g^{-1}(x) \text {. }
$$

The action of $\gamma \in \Gamma$ on the connection form $\alpha \in \mathcal{A}$ will be denoted by $\gamma(\alpha)$.

With any connection form $\alpha$ (i.e. with any connection $A$ ) we can associate a gauge isotropy subgroup $\Gamma_{\alpha}$,

$$
\Gamma_{\alpha}=\{\gamma \in \Gamma: \gamma(\alpha)=\alpha\}
$$

It is well known [22] that, with $p_{0} \in P$ any reference point in $P$ and $x_{0}=\pi\left(p_{0}\right)$,

$$
\Gamma_{\alpha}=\left\{\gamma \in \Gamma: \nabla^{\alpha}(\gamma)=0, g\left(x_{0}\right) \in C_{G}\left[H_{\alpha}\left(p_{0}\right)\right]\right\},
$$


where $H_{\alpha}\left(p_{0}\right)$ is the holonomy group of the connection $\nabla^{\alpha}$ at $p_{0}$, and $C_{G}[H]$ the centralizer of $H$ in $G$, i.e.

$$
C_{G}[H]=\{g \in G:[g, h]=0 \quad \forall h \in H\} .
$$

Thus, $\Gamma_{\alpha}$ is isomorphic to a subgroup of the compact group $G$; notice that the isomorphism, given by $\nabla^{\alpha} \gamma=0$, depends on $A$.

Remark 1. We stress, in view of later discussion, that for the null connection $A^{0}, H_{0}\left(p_{0}\right)=$ $\{e\}$ for all points $p_{0} \in P$, and thus $\Gamma_{0}$ is the group of covariantly constant (along $A^{0}$ ) functions from $B$ to $G$, i.e. $\Gamma_{0} \simeq G$. $\odot$

Given a connection $\alpha$ with isotropy group $\Gamma_{\alpha}$ we can consider: (i) the fixed space of $\Gamma_{\alpha}$, i.e. the space of connections (associated with connection forms) which are left invariant by $\Gamma_{\alpha}$,

$$
\mathcal{F}(\alpha)=\left\{\beta \in \mathcal{A}: \gamma(\beta)=\beta \quad \forall \gamma \in \Gamma_{\alpha}\right\}=\left\{\beta: \Gamma_{\alpha} \subseteq \Gamma_{\beta}\right\} ;
$$

and (ii) the isotropy type of $\alpha$, i.e. the space of connections (associated with connection forms) having isotropy subgroups which are $\Gamma$-conjugated to that of $\alpha$,

$$
\Sigma(\alpha)=\left\{\beta \in \mathcal{A}: \exists \gamma \in \Gamma: \Gamma_{\beta}=\gamma \Gamma_{\alpha} \gamma^{-1}\right\}
$$

The orbit of $\alpha$ under $\Gamma$ will be denoted by $\Gamma(\alpha)$ (or, for ease of notation, by $\omega_{\alpha}$ ). If we consider the gauge transformed of $\alpha$, we have easily that $\Gamma_{\gamma(\alpha)}=\gamma \Gamma_{\alpha} \gamma^{-1}$, or in other words

$$
\Gamma(\alpha) \subseteq \Sigma(\alpha)
$$

thus, the equivalence classes under the relation of belonging to the same isotropy type consist of (necessarily, disjoint) unions of gauge orbits.

Actually, this equivalence relation leads to a stratification of $\mathcal{A}$ [22] (see [27-29] for more detail), pretty much as in the classical case of compact group action on finite dimensional manifolds; however, we have now a countable - rather than finite - set of strata [26-29].

The set $\Sigma(\alpha)$ will be called the stratum of $\alpha$, and it can be shown to be a smooth manifold, and actually a principal bundle [29].

\section{Sobolev norms, and slices}

Let us now consider $\mathcal{A}$ in some more detail. We have already noticed that it is a linear space; using the $G$-invariant scalar product in $\mathcal{G}$, denoted by $\langle.,$.$\rangle , we can define a scalar$ product in $\mathcal{A}$ by

$$
(\alpha, \beta)=\int_{B}\left[\sum_{\mu=1}^{n}\left\langle A_{\mu}(x), B_{\mu}(x)\right\rangle\right] \mathrm{d}^{n} x
$$


where $\alpha=A_{\mu} \mathrm{d} x^{\mu}, \beta=B_{\mu} \mathrm{d} x^{\mu}$. Let $|\alpha|=(\alpha, \alpha)^{1 / 2}$ denote the corresponding norm. Fixing a connection $C^{0} \in \mathcal{C}$ and using the induced covariant derivative $\nabla^{0}$ we define a Sobolev scalar product and a Sobolev norm of class $k$ on $\mathcal{A}$ by

$$
(\alpha, \beta)_{k}=\sum_{j=0}^{k}\left[\int_{B}\left(\left(\nabla^{0}\right)^{j} \alpha,\left(\nabla^{0}\right)^{j} \beta\right) d^{n} x\right]
$$

and

$$
\left.\|\alpha\|_{k}=\left.\left(\sum_{j=0}^{k} \int_{B} \mid\left(\nabla^{0}\right)^{j}\right) \alpha\right|^{2} d^{n} x\right)^{1 / 2} .
$$

We will call $\mathcal{A}_{k}$ the completion of $\mathcal{A}$ with respect to this norm. Then $\mathcal{A}_{k}$ is an Hilbert space, and different choices of the connection $C^{0} \in \mathcal{C}$ give rise to equivalent norms [30-33]. If we consider also the Sobolev completion $\Gamma_{k}$ of the gauge group $\Gamma$, we have that, for $k>k_{0}=[(\operatorname{dim}(B)+1) / 2], \Gamma_{k}$ is an infinite dimensional Hilbert-Lie group modelled on a separable Hilbert space (this same condition does also ensure that the Sobolev norm dominates the $L_{\infty}$ one, see e.g. [34]). The action of $\Gamma$ on $\mathcal{A}$ can be extended to a smooth action of $\Gamma_{k}$ on $\mathcal{A}_{k-1}$ and the $\Gamma_{k}$ orbits are closed in $\mathcal{A}_{k-1}[25,35,36]$

From now on we will assume that all objects requiring Sobolev completion have been completed in appropriate norms and we will write again $\mathcal{A}$ and $\Gamma$ instead of $\mathcal{A}_{k}$ and $\Gamma_{k+1}$, and $(\alpha, \beta)$ instead of $(\alpha, \beta)_{k}$.

We remark that the norm (13) induce a $\Gamma$-invariant distance $d$ on $\mathcal{A}$ defined in the usual way, i.e. as $d(\alpha, \beta)=\|\alpha-\beta\|$.

It is known $[22,23,26,27,31,36]$ that the action of $\Gamma$ on $\mathcal{A}$ admits a slice $S_{\alpha}$ at any point $\alpha \in \mathcal{A}$. The existence of a slice guarantees the existence of a tubular neighbourhood $\mathcal{U}_{\alpha}$ of the $\Gamma$ orbit $\omega_{\alpha}$; this can be obtained by $\Gamma$-transporting $S_{\alpha}$, i.e. $\mathcal{U}_{\alpha}=\Gamma\left(S_{\alpha}\right)$.

We recall that a slice at $\alpha$ is a submanifold $S_{\alpha} \subseteq \mathcal{A}$ such that $\alpha \in S_{\alpha}$ and:

(i) $S_{\alpha}$ is transversal and complementary to the orbit $\omega_{\alpha}$ in $\mathcal{A}$ at $\alpha$;

(ii) $S_{\alpha}$ is trasversal to all the $\Gamma$ orbits which meet $S_{\alpha}$;

(iii) $S_{\alpha}$ is (globally) invariant under $\Gamma_{\alpha}$;

(iv) For $\beta \in S_{\alpha}$ and $\gamma \in \Gamma, \gamma(\beta) \in S_{\alpha}$ implies $\gamma \in \Gamma_{\alpha}$, i.e. $\Gamma_{\alpha}$ is the maximal subgroup which leaves $S_{\alpha}$ globally invariant; this also implies $\Gamma_{\beta} \subseteq \Gamma_{\alpha}$.

For later reference, we rewrite (i) as

$$
\mathrm{T}_{\alpha} \mathcal{A}=\mathrm{T}_{\alpha} S_{\alpha} \oplus \mathrm{T}_{\alpha} \omega_{\alpha}
$$

we also denote, again for later reference,

$$
S_{\alpha}^{0}=S_{\alpha} \cap \mathcal{F}(\alpha)
$$


It follows from properties (iii) and (iv) of $S_{\alpha}$, and the compactness of $\Gamma_{\alpha}$, that

$$
\begin{gathered}
S_{\alpha}^{0}=\Sigma(\alpha) \cap S_{\alpha}, \\
\Sigma(\alpha) \cap \mathcal{U}_{\alpha}=\bigcup_{\beta \in \omega_{\alpha}} S_{\beta}^{0} ;
\end{gathered}
$$

hence, from (10),(14) and (17), we have that

$$
\mathrm{T}_{\alpha} \Sigma(\alpha)=\mathrm{T}_{\alpha} \omega_{\alpha} \oplus \mathrm{T}_{\alpha} S_{\alpha}^{0} .
$$

\section{Michel theorem for pure gauge theories}

Using the notation defined above, we introduce the following two definitions:

Definition 1: A gauge orbit $\omega_{\alpha}$ is isolated in its stratum if and only if $\mathcal{U}_{\alpha} \cap \Sigma(\alpha)=\omega_{\alpha}$.

Definition 2: The $\Gamma$-orbit $\omega \subset \mathcal{A}$ is critical if points on $\omega$ are critical for any smooth $\Gamma$-invariant functional on $\mathcal{A}$.

We will prove that these are actually equivalent; that is, we have the:

Theorem. A gauge orbit $\omega$ is critical if and only if it is isolated in its stratum.

Sketch of the proof. Let us now consider a $\Gamma$-invariant functional $\mathcal{L}: \mathcal{A} \rightarrow R$ of class $C^{1}$ (a special case of this is the Yang-Mills functional), i.e. a functional such that

$$
\mathcal{L}(\gamma(\alpha))=\mathcal{L}(\alpha) \quad \forall \gamma \in \Gamma, \forall \alpha \in \mathcal{A} ;
$$

its differential at $\alpha, \mathrm{d} \mathcal{L}_{\alpha}: \mathrm{T}_{\alpha} \mathcal{A} \rightarrow R$, will be a linear and continuous operator; as $\mathcal{A}$ is a Hilbert space, this will correspond to an element $\phi_{\alpha} \in \mathrm{T}_{\alpha} \mathcal{A} \simeq \mathcal{A}$, such that $\mathrm{d} \mathcal{L}_{\alpha}(\beta)=$ $\left(\phi_{\alpha}, \beta\right)$.

It is easy to see that the invariance of $\mathcal{L}$ implies

$$
\phi_{\gamma(\alpha)}=\gamma\left(\phi_{\alpha}\right) .
$$

This also implies that if $\gamma \in \Gamma_{\alpha}$, then $\mathrm{d} \mathcal{L}_{\alpha}$ is invariant under $\gamma$; that is,

$$
\phi_{\alpha} \in \mathrm{T}_{\alpha} \mathcal{F}(\alpha) \text {. }
$$

On the other side, it is clear from (19) that $\left(\phi_{\alpha}, \xi\right)=0$ for all $\xi \in \mathrm{T}_{\alpha} \omega_{\alpha}$; thus we conclude [see (14),(18)] that

$$
\phi_{\alpha} \in \mathrm{T}_{\alpha} S_{\alpha}^{0} .
$$


It is clear from (19) that if $\alpha \in \mathcal{A}$ is a critical point for $\mathcal{L}$, all the points $\beta \in \Gamma(\alpha)$ are also critical for $\mathcal{L}^{2}$, which justifies definition 2 . The above discussion shows that if an orbit is isolated in its stratum, then it is critical.

We could also prove the converse, i.e. that if an orbit is critical, then it is isolated in its stratum; the proof of this would be just the same as the one given by Michel [1] for compact groups and finite dimensional manifolds, and thus is omitted.

From the above discussion we conclude that for functionals defined on the space of connections of a principal bundle (in Physics' language, pure gauge theories) we have - in the framework, with the definitions, and under the conditions introduced so far - the extension of Michel's theorem given above. $\odot$

Remark 2. The simplest nontrivial case where we have nontrivial (that is, not pure gauge) critical gauge orbits is provided by $G=S U(3)$. An analysis of strata in this case is provided by [29]; the bordering relations among strata are analyzed in [37]. $\odot$

\section{Theories with matter fields}

In physical application of gauge theories [39-44], one wants to consider not only pure gauge theories, but theories with matter fields as well. It turns out that our main result can be extended to this case as well, and actually that this extension does not present any new difficulty with respect to the pure gauge case; thus we sketch here such an extension, without repeating details already discussed above in the pure gauge frame.

In the case of theories with matter fields, together with the $G$-principal bundle $P$ over $B$, and the set $\mathcal{C}$ of connections on $P$ (see sect.1), we should consider a vector bundle $Q$ over the same base space $B$, having $G$ as structure group; the fiber of $Q$ will be a vector space $E^{q}$ (usually $\mathbf{R}^{q}$ or $\mathbf{C}^{q}$ ), and the set of differentiable sections of $Q$ will be denoted as $\mathcal{V}$. Notice this is not a complete space, and moreover that we should impose, as for the gauge fields, a finite energy condition (these remarks will naturally lead to consider a Sobolev space of fields in $\mathcal{V}$, see below).

By choosing an orthonormal frame in $E^{q}$, and using the reference connection chosen in $\mathcal{C}$ to transport this to the fiber over any point $x \in B$, a section $f \in \mathcal{V}$ is described in local coordinates by $\phi: B \rightarrow E^{q}$, i.e. by $q$ fields $\phi^{j}(x), j=1, \ldots, q, \phi^{j}: B \rightarrow E$; these are usually called matter fields.

The group $G$ acts on $E^{q}$ by a linear representation $T$, and we denote by $T_{g}$ or $T(g)$ the linear operator on $E^{q}$ corresponding to $g \in G$.

2 It is then natural to consider as index of $\alpha$ [38] its index as a critical point of the restriction of $d \mathcal{L}_{\alpha}$ to the slice $S_{\alpha}$, or equivalently to $S_{\alpha}^{0}$. 
We should then consider the sum bundle $P \oplus Q$ over $B$; we denote the space of sections of this by $\Gamma \oplus \mathcal{V}$, and a section by $\gamma \oplus f$.

An element $\gamma$ of $\Gamma$ expressed in local coordinates as $g(x)$ acts on $f \in \mathcal{V}$ - expressed in local coordinates as $\phi(x)$ - by

$$
[\gamma(f)](x)=T_{g(x)} \phi(x) .
$$

This means in particular that the gauge isotropy subgroup of $f$ is

$$
\Gamma_{f}=\left\{\gamma \in \Gamma: g(x) \in G_{\phi(x)} \quad \forall x \in B\right\} ;
$$

notice that this is in general a non-compact group.

We can define a natural scalar product in $\mathcal{V}$ using the scalar product $\langle.,$.$\rangle defined in E^{q}$ : indeed, we define

$$
(f, h)=\int_{B}\langle\phi(x), \chi(x)\rangle \mathrm{d}^{n} x
$$

where $f \simeq \phi(x), h \simeq \chi(x)$.

We can then proceed as in section 2, and define - using a connection $C^{0}$ and the induced covariant derivative - a Sobolev scalar product and a Sobolev norm of class $k$ on $\mathcal{V}$; this $k$ will be the same as that chosen in the analysis of connections. We will then denote by $\mathcal{V}_{k}$ the completion of $\mathcal{V}$ with respect to this norm, and consider from now on this set of sections (and drop - as it was already done for $\mathcal{A}$ - the subscript, for ease of notation).

Results concerning Hilbert-Lie group structures, and existence of slices and tubular neighbourhoods in $\mathcal{V}$, are described e.g. in [45].

Rather than repeating in $\mathcal{V}$ the analysis conducted in $\mathcal{A}$ - which would actually present some serious difficulty, as now we have non-compact isotropy subgroups - we will consider directly $\mathcal{A} \oplus \mathcal{V}$. The advantage of this follows from

Lemma. For any $\alpha \oplus f \in \mathcal{A} \oplus \mathcal{V}, \Gamma_{\alpha \oplus f}$ is compact.

Proof. This is a consequence of a very simple observation, i.e. that

$$
\Gamma_{\alpha \oplus f}=\Gamma_{\alpha} \cap \Gamma_{f} .
$$

Thus, $\Gamma_{\alpha \oplus f}$ is necessarily isomorphic to a (compact) subgroup of the compact group $G$, since this is the case for $\Gamma_{\alpha}$ (see sect.1). $\odot$

As a consequence, we can define strata in $\mathcal{A} \oplus \mathcal{V}$. We have seen above that we can also define a Sobolev metric, and thus neighbourhoods in $\mathcal{A} \oplus \mathcal{V}$ are well defined.

Remark 3. It should be stressed that we cannot proceed by defining strata in $\mathcal{V}$ and then "composing" them with strata in $\mathcal{A}$; the obstacles to this - due essentially to the noncompact nature of $\Gamma_{f}$ - are discussed e.g. in $[19,20]$ (these do also provide further detail on $\mathcal{V})$. $\odot$ 
We can then proceed as in sect.3, arriving at the same conclusions. In particular, we define $\mathcal{U}_{\alpha \oplus f}^{\varepsilon}$ as the set of sections $\beta \oplus h$ of $\mathcal{A} \oplus \mathcal{V}$ such that $\|\alpha-\beta\|+\|f-h\|<\varepsilon, \omega_{\alpha \oplus f}$ as the $\Gamma$-orbit of $\alpha \oplus f$, and $\Sigma(\alpha \oplus f)$ as the stratum of $\alpha \oplus f$ in $\mathcal{A} \oplus \mathcal{V}$. We say then that $\omega_{\alpha \oplus f}$ is isolated in its stratum if there is an $\varepsilon>0$ such that $\mathcal{U}_{\alpha \oplus f}^{\varepsilon} \cap \Sigma(\alpha \oplus f)=\omega_{\alpha \oplus f}$, and that $\omega_{\alpha \oplus f}$ is critical if points on $\omega_{\alpha \oplus f}$ are critical for any $\Gamma$-invariant smooth functional defined on $\mathcal{A} \oplus \mathcal{V}$.

We have then - as already mentiond, just following the procedure of section 3 , and thus the original proof by Michel [1] - that the orbit $\omega_{\alpha \oplus f}$ is critical if and only if it is isolated in its stratum.

\section{General gauge-equivariant evolution equations}

In the classical case (compact group $G$ acting on a finite dimensional manifold $M$ ), it is well known that the Michel's theory and its symmetry-based approach can be extended to consider general equivariant dynamics rather than just variational problems; in this way one can re-obtain ${ }^{3}[46,47]$ in particular the Equivariant Branching Lemma [48-50] (and its extension to the Hopf case) and the Reduction Lemma [51-53] (see also [54]); most of the results in equivariant bifurcation theory [52-58] are based on these lemmata [52].

The same holds here, i.e. one could obtain the corresponding results for equivariant vector fields on $\mathcal{A}$. Indeed, in this case equivariance means that

$$
X(\gamma(\alpha))=\gamma^{*}[X(\alpha)] \quad \forall \gamma \in \Gamma, \forall \alpha \in \mathcal{A},
$$

where $\gamma^{*}$ denote the action of $\Gamma$ on $\mathrm{T} \mathcal{A}$ induced by the action of $\Gamma$ on $\mathcal{A}$. Thus, for $\gamma \in \Gamma_{\alpha}$ and $X$ an equivariant vector field on $\mathcal{A}$, we have

$$
\gamma^{*}[X(\alpha)]=X(\gamma(\alpha))=X(\alpha) .
$$

We have thus proven the lemma below:

Lemma. Let $X: \mathcal{A} \rightarrow \mathrm{T} \mathcal{A}$ be a vector field on $\mathcal{A}$, equivariant under the gauge group $\Gamma$; then, $X(\alpha) \in \mathrm{T}_{\alpha} \mathcal{F}(\alpha)$.

3 It should be stressed that these results were originally obtained with no use of Michel's theory, and some years after the original Michel's paper. This seems to mean that on the one side mathematicians were not aware of the work of physicists on symmetry breaking, and on the other side also that physicists were not able to realize the relevance of the results they knew for other - not so far - fields (a partial exception being provided by [48]), i.e.

for general equivariant Nonlinear Dynamics. Surely, this fact points out a regrettable lack of communication between the two communities. 
From this lemma one could obtain immediately "infinite dimensional versions" of the Equivariant Branching Lemma and of the Reduction Lemma, in essentially the same way as in the finite dimensional case: indeed, the relevant feature here is the invariance under an equivariant flow (which includes the gradient flow for an invariant potential or functional) of the closure of a subspace defined by invariance properties (the strata or even the spaces $\mathcal{F}(\alpha)$ considered above). As $\mathcal{F}(\alpha)$ is in the closure of $\Sigma(\alpha)$, we also have immediately the

Corollary. Let $X: \mathcal{A} \rightarrow \mathrm{T} \mathcal{A}$ be a vector field on $\mathcal{A}$, equivariant under the gauge group $\Gamma$; then, $X(\alpha) \in \mathrm{T}_{\alpha} \Sigma(\alpha)$.

Remark 4. It should be stressed that the limitation to gauge theories and gauge invariant functionals (or, in this last lemma, gauge equivariant vector fields) was only dictated by the physical interest of this case; it turns out that one could as well extend Michel theory to the infinite dimensional setting (under suitable technical conditions) irrespective of the gauge structure. In this way, and using the symmetry theory for differential equations [5963], one could e.g. identify functions which are solutions for all the differential equations having a given symmetry (in a precise sense, amounting to commuting flows in a generalized function space) [64]. $\odot$

Remark 5. It is maybe also appropriate to stress, in this respect, that although the results based on $\mathcal{F}(\alpha)$ are, in principle, stronger than those based on $\Sigma(\alpha)$ (because the partition into sets $\mathcal{F}(\alpha)$ is finer ${ }^{4}$ than the one into strata $\Sigma(\alpha)$ ), from the physical point of view it is preferable, and more natural, to work with strata: on the one side, this mantains the identification among gauge-equivalent objects; and on the other side this permits to pass to $\mathcal{A} / \Gamma$ (also called the configuration space in physical literature), which is natural for a number of physical considerations.

On the other side, from a mathematical point of view one can be well justified in adopting the $\mathcal{F}(\alpha)$ point of view, as this is in general more powerful. This is quite similar to the situation in the finite dimensional case: from the physical point of view, the Michel approach is "the" natural one, as the orbit space $\Omega=M / G$ is the interesting one, and the approach based on the $\mathcal{F}(\alpha)$ cannot be applied to $\Omega$ if not passing through consideration of the strata. However, in other frames, e.g. in bifurcation theory [52,53] and anyway when one is interested in $M$ and not so much in $\Omega$, the stronger reduction provided by the invariance of $\mathcal{F}(\alpha)$ proves very useful as it allows a reduction to smaller dimensional submanifolds. $\odot$

4 More precisely, to speak about a finer partition we should consider the closures of strata, since - as already remarked - with our definition the $\mathcal{F}(\alpha)$ belong to the closure of $\Sigma(\alpha)$, not necessarily to $\Sigma(\alpha)$ itself. 


\section{Reduction and symmetry breaking.}

In physical applications of gauge theories, the theory - i.e. the functional $\mathcal{L}$ to be extremized - can depend on a control parameter, and one is specially interested in the occurrence of spontaneous symmetry breaking, i.e. in the case where $A^{0}$ is a critical point for all value of the control parameter $\lambda$, and a minimum for $\lambda<\lambda_{0}$, but for $\lambda>\lambda_{0}$ it is not a minimum and is thus unstable; minima are then realized by other, symmetry-breaking, critical points; we call these $A^{*}(\lambda)$ and denote the connection forms by $\alpha(\lambda)$.

We will consider a second order phase transition, i.e. the case where we will have

$$
\lim _{\lambda \rightarrow \lambda_{0}^{+}} A^{*}(\lambda)=A^{0} .
$$

In mathematical language, this corresponds to a bifurcation, and we have a continuous branch of bifurcating solutions.

Typically, these $A^{*}(\lambda)\left(\lambda>\lambda_{0}\right)$ have a symmetry which is less than the one of $A^{0}$ (this is invariant under any constant section, see remark 1 above), but which is the same for a whole set of values of $\lambda$ : thus, $A^{*}(\lambda)$ for $\lambda \in\left(\lambda_{0}, \lambda_{1}\right)$ belong to the same stratum. This also means that the $A^{*}(\lambda)$ correspond to gauge orbits which cannot be isolated in their stratum, so that the gauged version of Michel theorem given above cannot be used.

However, we can still be able to obtain relevant informations by "quotienting out" the unavoidable - and thus, in a sense, trivial - degeneracy of $\Gamma_{\alpha(\lambda)}$ in the direction "along the bifurcating branch". The idea will be essentially to separate the variation in this direction (on which symmetry considerations can be of no use) and the one in the other, transversal, directions ${ }^{5}$.

Thus we suppose that there is a stratum $\mu(\lambda)$ (notice this can depend on $\lambda$ ) in $\mathcal{A}$ such that $A^{0}$ belongs to the border of $\mu(\lambda)$ for all $\lambda \in\left(\lambda_{0}, \lambda_{1}\right)$, see above, and that $\mu(\lambda) / \Gamma$ is a one-dimensional manifold (the latter one is a strong assumption, which presumably could be somewhat relaxed).

We are then guaranteed of the vanishing of $(\delta \mathcal{L})[\alpha]$ in directions transversal to $\mu(\lambda)$; this means that in this case we can split the search for critical points of the $\Gamma$-invariant functional $\mathcal{L}$ in two steps: (i) we determine strata $\mu(\lambda)$ such that $\mu(\lambda) / \Gamma$ is one-dimensional; (ii) once we have determined such a $\mu(\lambda)$, we consider the restriction of $\mathcal{L}$ to $\mu(\lambda)$, and actually to $\mu(\lambda) / \Gamma$ : i.e. we reduce to a variational problem in one dimension.

Notice that when the functional is confining (i.e. we are guaranteed of the existence of a ball $\mathcal{B}$ in $\mathcal{A}$ such that $\mathcal{L}>\mathcal{L}\left[A^{0}\right]$ on $\mathcal{A} \backslash \mathcal{B}$ and $\delta \mathcal{L}$ is inward on $\partial \mathcal{B}$ ), we are guaranteed of

5 Notice that the application of Michel theorem to $G=S U(3)$ met the same problem; in that case, the direction "along the branch" would simply be the radial one, and thus one would simply consider the unit sphere in $\mathcal{G}=s u(3)$. 
the existence of a critical point in $\Sigma(\alpha)$, and when we add the condition that $A^{0}$ is unstable for $\lambda>\lambda_{0}$, we are actually guaranteed of the existence of critical gauge orbits in $\mu(\lambda)$.

This approach can be seen as an application of the "Symmetric Criticality Principle" of Palais [65,66]; or also - more simply - as a generalization of the equivariant branching lemma [48-50], well known in equivariant bifurcation theory [52-58].

Remark 6 . The symmetric criticality principle can also be applied - and is actually originally formulated [65] - in what we called the $\mathcal{F}(\alpha)$ frame. In this way, it becomes even more powerful for what concerns general gauge-equivariant evolution problems. However, the same considerations presented in remark 5 apply here, i.e. in the context of physical gauge theories one should identify gauge-related spaces and thus work with strata rather than with fixed spaces. $\odot$

\section{Extensions, generalizations, and discussion.}

We would like to conclude this note by a series of remarks concerning possible extensions of our results, comparison of these with existing results on critical points of gauge functionals, and/or comparison with the Michel theory for the classical case (we recall by this we mean the finite dimensional case, i.e. a compact Lie group $G$ acting on a finite dimensional smooth manifold $M$ ).

Remark 7. First of all, we notice that in the classical case, one can apply the classical tools of variational analysis (e.g. Lyusternik-Shnirelman and Morse theories; see e.g. [67] and refernces therein, and [68]), and also stratified Morse theory [69] to obtain further information on the number and nature of critical points in (the closure of) each stratum; see e.g. [70] for a simple example, and especially [71] for a recent - physically relevant and mathematically more interesting - application of this approach. It appears that the same holds - with increased technical difficulties to be expected, as we are now in an infinite dimensional setting - for our present extension. $\odot$

Remark 8. We would also like to mention that in the classical case one is able, modulo some further assumption on the $G$-action on $M$ - to project an equivariant vector field onto a field in the orbit space ${ }^{6} \Omega=M / G$. In the present setting, it is known [22,29] that $\mathcal{A}$ can be decomposed as the union of principal $G_{j}$-bundles (the $G_{j}$ being certain subgroups of $G)$; moreover, we know that $\mathcal{A}$ has a proper isotropy stratification. Thus it would be worth studying if there are reasonable conditions under which any equivariant vector field

6 Notice that in general $\Omega$ is not a manifold, but it is a stratified manifold, i.e. the union of strata in the geometric (Whitney) sense, which themselves are manifolds. Notice also that our theorem guarantee that the projected field will be tangent to these strata, provided one proves the Whitney strata are union of Michel strata (see $[46,47]$ ). 
$\phi^{*}: \mathcal{A} \rightarrow \mathrm{T} \mathcal{A}$ can be projected to some vector field $\psi^{*}: \mathcal{A} / \Gamma \rightarrow \mathrm{T}(\mathcal{A} / \Gamma)$ (wherever this makes sense). $\odot$

Remark 9. In this note we adopted the point of view of studying the singular strata (i.e. orbits which are not in the generic stratum, or equivalently reducible connections), arguing that - as it is shown by our extension of Michel theorem - these will provide "generic" solutions in the presence of symmetry, precisely because they are singular, or even better "as singular as possible".

A different approach is also possible, using again the stratification but proceeding the other way round, i.e. considering first the generic orbits (i.e. $\Gamma_{A} \simeq G$ ), then connections which are reducible but with large $\Gamma_{A}$, and so on, and at each stage restricting attention to these "minimally reducible" connections, on which one can, by the procedure very shortly described in a moment, apply the results holding for reducible connections $[22,23]$ provided the theory is set on a reduced bundle [29]. Let us consider a reducible connection $\alpha$; from (5), the $\Gamma_{\alpha}$ would depend on the reference point

$$
p_{0}=\left(x_{0}, \gamma\left(x_{0}\right)\right) \in \pi^{-1}\left(x_{0}\right) \subset P ;
$$

choosing a different reference point $g p_{0}$ on the same fiber will change $H_{A}\left(p_{0}\right)$ to $H_{A}\left(g p_{0}\right)=$ $g H_{A}\left(p_{0}\right) g^{-1}$, and it follows easily that $C_{G}\left[H_{A}\left(g p_{0}\right)\right]=g\left(C_{G}\left[H_{A}\left(p_{0}\right)\right]\right) g^{-1}$. Thus, nothing changes - in $H_{A}$ and hence in $\Gamma_{\alpha}$ - when we act by

$$
g \in J_{A} \equiv C_{G}\left[C_{G}\left[H_{A}\left(p_{0}\right)\right]\right] .
$$

We can then consider a subbundle $P_{A}$ over the same base space $B$ and fiber $\pi^{-1}(x) \simeq$ $J_{A} \subseteq G$; the connection $\alpha$ is irreducible over this subbundle. We can thus analyze more and more singular strata in $\mathcal{A}$ by proceding along chains of subbundles [29]; strata can be analyzed in terms of these, and bordering relations can be expressed as relations among characteristic classes [37]. This approach could also be extended to gauge theories with matter fields. $\odot$

Remark 10. At the same time as the Michel-Radicati approach [1-4], a different approach, also based on geometry of group action, was proposed by Cabibbo and Maiani [72]; this is not equivalent to the Michel-Radicati approach, but seems to somewhat be a precursor of the symmetric criticality principle of Palais [65] mentioned above. $\odot$

Remark 11. In the present note, we have only mentioned those results on the geometry of the gauge orbit space (configuration space) $\mathcal{A} / \Gamma$ that we needed; however, the geometry of this space has been studied quite in detail. We refer to [22-30,35,37], already mentioned, and e.g. to [73-77] for further detail and bibliographic indications. $\odot$

Remark 12. It should be stressed (see also the remarks below) that the results presented here - and the method they provide to search for critical points of gauge-invariant functionals - are settled in general terms, i.e. for general (smooth) gauge-invariant functionals; this is in principles an advantage of them. However, this fact can also be considered as 
a weakness of our approach: that is, in this way we fail to take advantage of the specific features of the functionals of interest in physical gauge theories (see the appendices). $\odot$

Remark 13. We would like to stress that the approach presented here is quite general, and can be applied to any base manifold $B$ and Lie group $G$; similarly, it can be used to look for any kind of critical point, and not only for minima and/or for selfdual or anti-selfdual critical points.

In this respect, we recall that the critical points which are not minima can be quite relevant physically: this is the case e.g. in the WKB theory (see also the Duistermaat-Heckman localization theory [78-80], guaranteeing that in certain cases the WKB approximation is exact).

Similarly, we recall that the celebrated results of Bourguignon and Lawson [31-33], stating that all weakly stable critical points of Yang-Mills functionals are self-dual or anti-self-dual, apply under precise conditions (which enclose the physically relevant cases); in particular, they only hold in dimension four, and for $G=S U(2)$ and $S U(3)$ [with extensions to $U(3)$ and $S O(4)$ ], and do not deal with unstable critical points.

Moreover, we also mention that the present approach could also be useful (as it localizes the critical points in strata and thus provides a natural parametrization of them) in determining the moduli space for instanton/monopole solutions, and the Atiyah-Hitchin metric. $\odot$

Remark 14. As already mentioned in the introduction, previous attempts to extend Michel theory to the gauge case exist, and were able to provide some results [19,20]; however, they suffered from severe limitations. Essentially, these only considered functionals on $\mathcal{V}$ (moreover, overlooking any contact structure $[59,62,75]$ when considering dependence on derivatives). These limitations followed from tackling the problem by the matter field sector (see the remarks in sect.4 for the difficulties with this approach); in the present complete - and geometrical - setting, it is the strong geometrical structure present in $\mathcal{A}$, i.e. in the pure gauge sector, which allows to tame the problems arising in $\mathcal{V}$ and which forced the previous attempts $[19,20]$ to such a limited scope. $\odot$

Remark 15. It should also be stressed that the results given here extend to the case of a gauge functional depending on higher order (covariant) derivatives, thus extending substantially the case of proper Yang-Mills functionals. $\odot$

Remark 16. More in general, apart from technical problems, the whole construction considered here, and by Michel theory, relies on the concept of group action on a manifold, and of invariant functionals (or equivariant ones once we consider e.g. the gradients), together with a suitable topology and an appropriate "agreement" between the manifold and group topologies (the requirement that group orbits are proper submanifolds of the manifold); it is quite clear that this is a very general situation, and thus that the reach of Michel theory is correspondingly ample. $\odot$ 
Thus, in conclusion, we believe Michel theory provides a new approach to a number of questions which are extremely relevant for Physics, and possibly also for Mathematics; needless to say, the credit for it should go to the original geometric view it embodies [1], and not to the simple, albeit useful, extension given here.

\section{Acknowledgements}

This work was started at the Mathematische Forschunginstitut in Oberwolfach; our staying in M.F.O. was supported by the Volkswagen Stiftung under the "Research in Pairs" program. We would like to thank the Director of M.F.O., prof. M. Kreck, for hospitality.

The work was completed during the stay of one of us (G.G.) in I.H.E.S.; warm thanks go to the Director, prof. J.P. Bourguignon, and all the personnel of the Institute for the invitation and hospitality.

The work of P.M. received essential support from GNFM-CNR through travel funds to Oberwol-

fach, Loughborough and Bures sur Yvette. We also benefited from travel grants by Loughborough University.

In the preparation of this work we have bothered several friends and colleagues with our questions; we would like in particular to thank C. Bachas and A. Chakrabarti. 


\section{Appendix A. Special solutions in theories with matter fields.}

In this note, we considered theories defined by any smooth gauge-invariant functional on $\mathcal{A}$ or on $\mathcal{A} \oplus \mathcal{V}$; however, in physical gauge theories - i.e. Yang-Mills theories [39-44] - one mostly meets functionals with a precise form.

In this and the following appendices we want to show how our results apply to proper Yang-Mills theories, in order to get special solutions of interest, and recover the MichelRadicati results [3,4] in the $\mathrm{SU}(3)$ case. In this first appendix we want to show how we can by our result get some special, and relevant, solutions in the case of theories with matter fields, once we have determined critical points of the pure gauge part of the theory.

Thus we will consider some vector space $E$ and a vector bundle over $B$ with fiber $E$ having $G$ as structure group. When we include matter fields $\phi: B \rightarrow E$ in the theory, we have restricting to the physically relevant situation - a functional

$$
\mathcal{L}=\mathcal{L}_{g}(A, \phi)+\mathcal{L}_{p}(\phi)+\mathcal{L}_{y m}(A),
$$

where the three parts of the functional are defined as

$$
\begin{aligned}
\mathcal{L}_{g} & =\int_{B}\left|\left(\nabla_{A} \phi\right)(x)\right|^{2} \mathrm{~d}^{n} x \\
\mathcal{L}_{p} & =\int_{B} V[\phi(x)] \mathrm{d}^{n} x \\
\mathcal{L}_{y m} & =\int_{B}\left|F_{\mu \nu}[A(x)]\right|^{2} \mathrm{~d}^{n} x
\end{aligned}
$$

with $V$ a smooth function (potential) $V: E^{q} \rightarrow R$, and the norms defined via the appropriate scalar products. We will consider $B=R^{n}$, and $A^{0}$ is the flat connection.

In looking for critical points of this functional, we can look for a special class of these, i.e. we look for functions $\phi(x)$ and $A_{\mu}(x)$ which extremize the three parts separately: these will then be a satisfactory extremizing solution for $\mathcal{L}$ (although not all the solutions will be obtained in this way).

We can start from $\mathcal{L}_{y m}$, and suppose we have determined a connection $\alpha$ (i.e. the functions $A_{\mu}(x)$, valued in $\mathcal{G}$ ) which is critical for $\mathcal{L}_{y m}$; in particular, these could be determined by means of our previous results.

Let us now consider $\mathcal{L}_{p}$; we consider $\mathcal{F}$, the set of sections (in suitable Sobolev class) of the bundle over $B$ having $E$ as fiber, and $M \subset E$ the set of points on the fiber on which $V$ has extremal points. We then define $\mathcal{F}_{M} \subset \mathcal{F}$ as the set of sections $\phi$ such that $\phi(x) \in M$ for all $x \in B$. It is clear that any $\phi \in \mathcal{F}_{M}$ is critical for $\mathcal{L}_{p}$.

We should now consider $\mathcal{L}_{g}$, the only part in which $A$ and $\phi$ interact: this will be extremal - actually minimal - if $\phi$ is covariantly constant along $\alpha$. We will try to build a $\psi$ which satisfy this condition and is in $\mathcal{F}_{M}$. 
Thus, we consider a reference point $x_{0} \in B$, and choose $\psi\left(x_{0}\right)=m_{0} \in M$; then we define $\psi(x)$ by the condition $\nabla_{A} \psi=0$, i.e. as the solution to the equation

$$
\frac{\partial \psi}{\partial x^{\mu}}=-A_{\mu}(x) \psi(x)
$$

To this end, we choose for any $x \in B$ a parametrized path $\xi(s)$ such that $\xi(0)=x_{0}$ and $\xi(1)=x$. Now, the required solution is provided by

$$
\psi(x)=g(x) m_{0}
$$

where $g(x)$ is defined as

$$
g(x)=-\int_{0}^{1} A_{\mu}[\xi(s)] \frac{\partial \xi^{\mu}}{\partial s} \mathrm{~d} s
$$

Clearly, this depends on the choice of the path $\xi(s)$ : that is, such $g(x)$ is defined up to an element of the holonomy group of $\alpha$ at the point $p=(x, g(x))$; this was previously noted as $H_{A}(p)$. Correspondingly, $\psi(x)$ is defined up to the action of $H_{A}(p)$ on $g(x)$ : thus $\psi(x)$ is well defined only if at any point

$$
H_{A}[x, g(x)] \subseteq G_{\psi(x)}
$$

By general results on $H_{A}$ along covariantly constant curves [82], this condition can be checked by looking at the fiber over $x_{0}$ alone; moreover, we have [22] that

$$
H_{A}\left(x_{0}, g\right)=g H_{A}\left(x_{0}, e\right) g^{-1}
$$

and thus we can just require that

$$
H_{A}\left(x_{0}, e\right) \subset G_{m_{0}} .
$$

It should be recalled [22] that a close relationship exists between the isotropy group $\Gamma_{\alpha}$ and the holonomy group $H_{A}$ : indeed,

$$
\Gamma_{\alpha}=\left\{\gamma \in \Gamma: \nabla_{A}(\gamma)=0 ; \gamma\left(x_{0}\right) \in C_{G}\left[H_{A}\left(p_{0}\right)\right]\right\}
$$

where $C_{G}(H)$ is the centralizer of $H \subseteq G$ in $G$, and $p_{0}=\left(x_{0}, g\right)$ a reference point in $\pi^{-1}\left(x_{0}\right) \subset P$.

In this way we have determined, given $A$, which extremal points $m \subset M$ are suitable for the construction described above. 


\section{Appendix B. Solutions associated to the null connection}

Let us further restrict the setting of appendix A; that is, consider the critical orbit in $\mathcal{A}$ corresponding to pure gauges, or in other words the gauge orbit $\omega_{0}=\Gamma\left(A^{0}\right)$. Notice that in order to be sure $\omega_{0}$ is isolated in its stratum, and thus critical, it suffices that the action of $\Gamma$ is free (so that only pure gauges have $\Gamma_{\alpha} \simeq G$ ), as it is usually the case in physical applications.

In this case, we have immediately

$$
\begin{gathered}
H_{A}\left(x_{0}, g\right)=\{e\} \quad \forall g \in G \\
\Gamma_{0}=\left\{\gamma \in \Gamma: \nabla^{0}(\gamma)=0\right\} \simeq G
\end{gathered}
$$

and also the $f$ given by the construction in appendix A will be covariantly constant along $\nabla^{0}$, i.e. $\phi^{j}(x)=$ const for any $j$ and on any chart on $B$.

Thus, for the corresponding matter fields, we know that we can restrict to consider

$$
\mathcal{V}_{0}=\left\{f \in \mathcal{V}: \nabla^{0}(f)=0\right\}
$$

or, in other words, we can simply consider $f\left(x_{0}\right)=\Phi$; notice that $\Gamma_{0}$ acts naturally on $\mathcal{V}_{0}$ and, with obvious notation,

$$
[\gamma(f)]\left(x_{0}\right)=g_{0} \Phi .
$$

We can thus apply the classical version of Michel theorem in order to determine the $f \in \mathcal{V}_{0}$ which are isolated in their stratum, and thus the results of [1-4] immediately apply to this case.

Notice that, to be completely rigorous, we should remove the radial degeneracy (indeed $f$ and $\lambda f$, with $\lambda \in R$, will have the same isotropy group) or equivalently consider "critical directions" (see sect.6). 


\section{Appendix C. The SU(3) case}

The motivation for Michel's theory was provided, as already mentioned in the introduction, by the $S U(3)$ theory of hadronic interactions $[3,4,7]$. In this case, the relevant group action is the adjoint representation of $S U(3)$; this acts on the space $\mathcal{M}$ of three-dimensional unitary traceless matrices, and thus on $R^{8}$ : an invariant potential is then a function $V: R^{8} \rightarrow R$ such that $V(g \cdot x)=V(x)$, the action of $g \in S U(3)$ on the matrix $M$ being given by $g: M \in R_{g} M R_{g}^{-1}$, where $R_{g}$ is the matrix representing $g$ in the standard three-dimensional representation of $S U(3)$. In this case $\Omega=\mathcal{M} / G$ is two dimensional.

One considers then the restriction of $V$ to the unit sphere $\mathcal{M}_{0}$ in $\mathcal{M}$ under the appropriate scalar product, i.e. the $G$-invariant scalar product in the algebra $s u(3)$; this is given by $(A$. $B)=(1 / 2) \operatorname{Tr}(A . B)$. One can then check $[3,4,53]$ that the points in $\mathcal{M}_{0} / G$ corresponding to physical particles (the $S U(3)$ octet) are isolated in their strata, and thus the associated $G$-orbits identify critical orbits for any invariant potential on $\mathcal{M}_{0}$, and hence directions of symmetry breaking for any invariant potential on $\mathcal{M}$. This shows that predicting the actual directions of symmetry breaking in hadronic interactions is not a virtue of any particular model, but only of its invariance properties [5,7].

We will now discuss in some detail the extension of this result to the complete gauge setting considered here; such an extension is actually immediate (so much that Michel and Radicati did not feel any need to discuss it) but we believe it can help in fixing the idea about our present result to see how this applies in a very well known case.

Let us consider the structure of SU(3) subgroups; this is given by the following diagram:

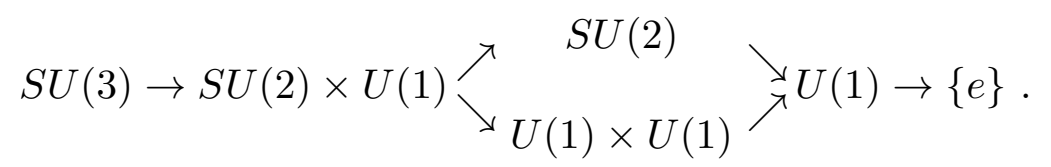

In the spirit of studying symmetry breaking from the trivial solution $(\alpha, f)=\left(A^{0}, 0\right)$ - and having already considered the case where symmetry is broken only in the matter sector, while that where it is broken only in the gauge sector is equivalent to the pure gauge case - we focus on $G_{0}=S U(2) \times U(1)$; thus, we consider critical gauge orbits in $\mathcal{A}$ with

$$
\Gamma_{A}=S U(2) \times U(1) \equiv G_{0} .
$$

For such a critical orbit - i.e. fixing a critical $A$ which satisfies (C2) - we have

$$
H_{A}\left(p_{0}\right)=Z\left(\Gamma_{A}\right)=U(1) \times U(1)=Z(G) .
$$

From the requirement that $H_{A} \subseteq G_{m_{0}}$ (i.e. in this case $H_{A} \subseteq G_{0}$ ), see (A9), we immediately reduce to three possibilities for $\Gamma_{f}^{(A)}=\left\{\gamma \in \Gamma_{A}: \gamma(f)=0\right\}$; i.e., this can be 
isomorphic to either $G$, either $G_{0}$, either $Z(G)$. In the first case we just have gauge fields (no symmetry breaking in the matter sector), while in the third case we would need further hypotheses to be sure the critical point located in the closure of the stratum is not actually on its border (and thus in the most singular stratum with isotropy type $G_{0}$ ). Thus, the case of interest is that of

$$
\Gamma_{f}^{(A)}=\Gamma_{(\alpha, f)}=S U(2) \times U(1) .
$$

The construction of appendix A tells how to build a section $f \in \mathcal{V}$ which is critical for (A1) in this case; more generally, define

$$
\mathcal{V}_{A}=\left\{f \in \mathcal{V}: \Gamma_{A} \cap \Gamma_{f}=\Gamma_{A}\right\}=\left\{f \in \mathcal{V}: \Gamma_{A} \subseteq \Gamma_{f}\right\}
$$

(this is analogous to the $\mathcal{V}_{0}$ considered above); we consider then, for $\omega_{\alpha}$ critical in $\mathcal{A}$, the restriction $\mathcal{L}_{A}$ of $\mathcal{L}: \mathcal{A} \oplus \mathcal{V}$ to $\alpha \oplus \mathcal{V}_{A} \simeq \mathcal{V}_{A}$; by the Symmetric Criticality Principle [65,66], the critical points of $\mathcal{L}_{A}$ will also be critical points of $\mathcal{L}$.

Within $\mathcal{V}_{A}$, we can proceed as for $\mathcal{V}_{0}$ in appendix $\mathrm{B}$, and thus use again the classical version of Michel theorem; once again, this is due to the fact that $\Gamma_{A}$ is canonically isomorphic to a compact subgroup of $G$.

Thus we conclude that for $\mathrm{SU}(3)$ (acting on $E^{q}$ by a free representation $T$ ) we always have, in the presence of symmetry breaking in both the pure gauge and the matter sectors, critical orbits $\omega_{\alpha, f}$ with the symmetry of both $\alpha$ and $f$ given by a group isomorphic to $S U(2) \times U(1)$; these correspond again to the critical directions determined by Michel and Radicati [1-4]. 


\section{References}

1. L. Michel, "Points critiques de fonctions invariantes sur une G-variété", Comptes Rendus Acad. Sci. Paris 272-A (1971), 433-436

2. L. Michel and L. Radicati, "Breaking of the $S U_{3} \times S U_{3}$ symmetry in hadronic Physics"; in M. Conversi (ed.), "Evolution of particle Physics (E.Amaldi Festschrift)", pp. 191-203, Academic Press 1970

3. L. Michel and L. Radicati, "Properties of the breaking of hadronic internal symmetry", Ann. Phys. (N.Y.) 66 (1971), 758-783

4. L. Michel and L. Radicati, "The geometry of the octet", Ann. I.H.P. 18 (1973), 185

5. L. Michel, "Nonlinear group action. Smooth action of compact Lie groups on manifolds", in "Statistical Mechanics and Field Theory", R.N. Sen and C. Weil eds., Israel University Press, Jerusalem 1971

6. L. Michel, "Les brisure spontanées de symétrie en physique", J. Phys. (Paris) 36 (1975), C7 41

7. L. Michel, "Symmetry defects and broken symmetry. Configurations. Hidden symmetry", Rev. Mod. Phys. 52 (1980), 617-651

8. M. Abud and G. Sartori, "The geometry of orbit space and natural minima of Higgs potentials", Phys. Lett. B 104 (1981), 147-152

9. M. Abud and G. Sartori, "The geometry of spontaneous symmetry breaking", Ann. Phys. 150 (1983), 307-372

10. G. Sartori and V. Talamini, "Universality in orbit spaces of compact linear groups", Comm. Math. Phys. 139 (1991), 559-588

11. G. Sartori and G. Valente, "Orbit spaces of reflection groups with 2,3 and 4 basic polynomial invariants", J. Phys. A 29 (1996), 193-223

12. G. Sartori, "Geometric invariant theory. A model-independent approach to spontaneous symmetry and/or supersymmetry breaking", Riv. N. Cim. 14 (1991) no. 11, 1-120

13. M.J. Field and R.W. Richardson, "Symmetry-breaking and the maximal isotropy subgroup conjecture for reflection groups", Arch. Rat. Mech. Anal. 105 (1989), 61-94

14. M.J. Field and R.W. Richardson, "Symmetry-breaking in equivariant bifurcation problems", Bull. A.M.S. 22 (1990), 79-84

15. M.J. Field and R.W. Richardson, "Symmetry-breaking and branching patterns in equivariant bifurcation theory - I", Arch. Rat. Mech. Anal. 118 (1992), 297-348

16. M.J. Field and R.W. Richardson, "Symmetry-breaking and branching patterns in equivariant bifurcation theory - II", Arch. Rat. Mech. Anal. 120 (1992), 147-190

17. G. Bredon, Compact transformation groups, Academic Press, 1972

18. M.J. Field, Symmetry breaking for compact Lie groups, Mem. A.M.S. 120 n.574 (1996), 1-170

19. G. Gaeta, "Michel's theorem and critical section of gauge functionals", Helv. Phys. Acta 65 (1992), 922-964

20. G. Gaeta, "Critical sections of gauge functionals: a symmetry approach"; Lett. Math. Phys. 28 (1993), $1-11$

21. G. Gaeta, "Equivariant branching lemma: dynamical systems, evolution PDEs, and gauge theories", Acta Appl. Math. 28 (1992), 43-68

22. I.M. Singer, "Some remarks on the Gribov ambiguity", Comm. Math. Phys. 60 (1978), 7-12

23. I.M. Singer, "The geometry of the oprbit space for non-abelian gauge theories" Physica Scripta 24 (1981), 817-820

24. M.S. Narashiman and T.R. Ramadas, "Geometry of SU(2) gauge fields" Comm. Math. Phys. 67 (1979), 121-136

25. G. Dell'Antonio and D. Zwanziger, "Every gauge orbit passes inside the Gribov horizon", Comm. Math. Phys. 138 (1981), 291-299

26. J.P. Bourguignon, "Une stratification de l'espace des structures riemanniennes", Comp. Math. 30 (1975), 1-41

27. W. Kondracki and P. Sadowski, "Geometric structure on the orbit space of gauge connections", J. Geom. Phys. 3 (1986), 421-434 
28. W. Kondracki and J.S. Rogulski, "On the stratificatrion of orbit space for the action of automorphisms on connections", Diss. Math. 250 (1986), 1-62

29. A. Heil, A. Kersch, N. Papadopolous, B. Reifenhauser and F. Scheck, "Structure of the space of reducible connexions for Yang-Mills theories", J. Geom. Phys. 7 (1990), 489-505

30. K.B. Marathe and G. Martucci, "The geometry of gauge fields", J. Geom. Phys. 6 (1989), 1-106

31. H.B. Lawson, The theory of gauge fields in four dimensions, A.M.S. (Providence) 1985

32. J.P. Bourguignon and H.B. Lawson, "Stability and isolation phenomena for Yang-Mills fields", Comm. Math. Phys. 79 (1981), 189-230

33. J.P. Bourguignon, H.B. Lawson and J. Simons, "Stability and gap phenomena for Yang-Mills fields", Proc. Natl. Acad. Sci. USA 76 (1979), 1550-1553

34. H. Brezis, Analyse fonctionelle, Masson, Paris 1983

35. P.K. Mitter and C.M. Viallet, "On the bundle of connections and the gauge orbit manifold in Yang-Mills theory", Comm. Math. Phys. 79 (1981), 457-472

36. J. Sniatycki, G. Schwarz and L. Bates, "Yang-Mills and Dirac fields in a bag, constraints and reduction", Comm. Math. Phys. 176 (1996), 95-115

37. C.J. Isham, "Space-time topology and spontaneous symmetry breaking", J. Phys. A. 14 (1981), 29432956

38. M.F. Atiyah and J.D.S. Jones, "Topological aspects of Yang-Mills theory", Comm. Math. Phys. 61 (1978), 97-118

39. C.N. Yang and R.L. Mills, "Conservation of isotopic spin and isotopic gauge invariance", Phys. Rev. 96 (1954), 191-195

40. E.S. Abers and B.W. Lee, "Gauge theories", Phys. Rep. 9 (1973), 1-141

41. T. Eguchi, P.B. Gilkey and A.J. Hanson, "Gravitation, gauge theories, and differential geometry", Phys. Rep. 66 (1980), 213-393

42. W. Drechsler and M.E. Mayer, "Fibre bundle techniques in gauge theories", Lect. Notes Phys. 67, Springer 1977

43. M. Daniel and C.M. Viallet, "The geometrical setting of gauge theories of Yang-Mills type", Rev. Mod. Phys. 52 (1980), 175-197

44. C.J. Isham, Modern differential geometry for physicists, World Scientific 1989

45. R.S. Palais and C.L. Terng, Critical point theory and submanifold geometry, Lect. Notes Math. 1353, Springer 1988

46. G. Gaeta, "A splitting lemma for equivariant dynamics", Lett. Math. Phys. 33 (1995), 313-320;

47. G. Gaeta, "Splitting equivariant dynamics", Nuovo Cimento B 110 (1995), 1213-1226

48. G. Cicogna, "Symmetry breakdown from bifurcation", Lett. Nuovo Cimento 31 (1981), 600-602

49. A. Vanderbauwhede, Local bifurcation and symmetry, Pitman (Boston) 1982

50. G. Cicogna, "A nonlinear version of the equivariant bifurcation lemma", J. Phys. A 23 (1990), L1339L1343

51. M. Golubitsky and I.N. Stewart, "Hopf bifurcation in the presence of symmetry", Arch. Rat. Mech. Anal. 87 (1985), 107-165

52. M. Golubitsky, D. Schaeffer and I. Stewart, Singularities and groups in bifurcation theory - vol. II, Springer (New York) 1988

53. G. Gaeta, "Bifurcation and symmetry breaking", Phys. Rep. 189 (1990), 1-87

54. P. Chossat and M. Koenig, "Characterization of bifurcations for vector fields which are equivariant under the action of a compact Lie group", C. R. Acad. Sci. (Paris) 318 (1994), 31-36

55. M. Field, "Equivariant dynamical systems", Bull. A.M.S. 76 (1970), 1314-1318

56. D. Ruelle, "Bifurcations in the presence of a symmetry group", Arch. Rat. Mech. Anal. 51 (1973), 136-152

57. D.H. Sattinger, Group theoretic methods in bifurcation theory, Lect. Notes Math. 762, Springer 1979

58. D.H. Sattinger, Branching in the presence of symmetry, SIAM (Philadelphia) 1984

59. P.J. Olver, Applications of Lie groups to differential equations, Berlin, Springer 1986

60. G.W. Bluman and S. Kumei, Symmetries and differential equations, Springer, Berlin 1989

61. H. Stephani, Differential Equations. Their solution using symmetry; Cambridge 1991

62. G. Gaeta, Nonlinear symmetry and nonlinear equations, Kluwer, Dordrecht 1994 
63. P.J. Olver, Equivariants, invariants, and symmetry, Cambridge 1995

64. G. Gaeta and P. Morando, "Commuting-flow symmetries and common solutions to differential equations with common symmetry"; Preprint mp-arc 96-618 1996

65. R.S. Palais, "The principle of symmetric criticality", Comm. Math. Phys. 69 (1979), 19-30

66. R.S. Palais, "Applications of the symmetric criticality principle in mathematical physics and differential geometry", in "Proceedings of the 1981 Shanghai symposium on differential geometry and differential equations", Gu Chaohao ed., Science Press, Beijing, 1984

67. A. Ambrosetti, Critical points and nonlinear variational problems, Memoires Soc. Math. France 49 (supplement to Bull. S.M.F. 120), S.M.F., Paris, 1992

68. R.S. Palais, "Morse theory on Hilbert manifolds", Topology 2 (1963), 299-340

69. M. Goresky and R. MacPherson, Stratified Morse theory, Springer 1988

70. G. Gaeta, "Counting symmetry breaking solutions to symmetric variational problems", Int. J. Theor. Phys. 35 (1996), 217-229

71. L. Michel, "Extrema des fonctions sur la zone de Brillouin, invariantes par le groupe de symétrie du cristal et le renversement du temps", C. R. Acad. Sci. Paris B-322 (1996), 223-230

72. N. Cabibbo and L. Maiani, "Weak interactions and the breaking of hadronic symmetry"; in M. Conversi (ed.), "Evolution of particle Physics (E. Amaldi Festschrift)", pp. 50-80, Academic Press 1970

73. S. Klimek, W. Kondracki, W. Oledzki and P. Sadowski, "The density problem for infinite dimensional group actions", Comp. Math. 68 (1988), 3-10

74. O. Babelon and C.M. Viallet, "The riemannian geometry of the configuration space of gauge theories", Comm. Math. Phys. 81 (1981), 515-525

75. P. Cotta-Ramusino and C. Reina, "The action of the group of bundle automorphisms on the space of connections and the geometry of gauge theories", J. Geom. Phys. 1 (1984), 121-155

76. M.C. Abbati, R. Cirelli and A. Manià, "The orbit space of the action of gauge transformation group on connections", J. Geom. Phys. 6 (1989), 537-558

77. M.C. Abbati, R. Cirelli, A. Manià and P. Michor, "The Lie group of automorphisms of a principal bundle", J. Geom. Phys. 6 (1989), 215-235

78. J.J. Duistermaat and G.J. Heckman, "On the variation in the cohomology in the symplectic form of the reduced phase space", Invent. Math. 69 (1982), 259

79. J.M. Bismut, "Localization formulas, superconnections, and the index theorem for families", Comm. Math. Phys. 103 (1986), 127

80. L.C. Jeffrey and F.C. Kirwan, "Localization and the quantization conjecture", Topology 36 (1997), 647-694

81. C. Ehresman, "Les prolongements d'une varieté differentiable, I-V", C. R. Acad. Sci. (Paris) 233 (1951), 598, 233 (1951), 777, 233 (1951), 1081, 234 (1952), 1028, 234 (1952), 1424

82. J.L. Koszul, Lectures on fibre bundles and differential geometry (Tata Institute), Springer 1986 